\title{
Japanese Cardiovascular Disease Patients with Diabetes Mellitus Suffer Increased Tooth Loss in Comparison to Those without Diabetes Mellitus-A Cross-sectional Study
}

\author{
Norio Aoyama ${ }^{1}$, Jun-ichi Suzuki ${ }^{2,3}$, Naho Kobayashi ${ }^{4}$, Tomoya Hanatani ${ }^{5}$, \\ Norihiko Ashigaki ${ }^{6}$, Asuka Yoshida ${ }^{4}$, Yuka Shiheido ${ }^{4}$, Hiroki Sato ${ }^{4}$, Hidetoshi Kumagai ${ }^{2}$, \\ Yuichi Ikeda ${ }^{7}$, Hiroshi Akazawa ${ }^{7}$, Issei Komuro ${ }^{7}$, Masato Minabe ${ }^{1}$, \\ Yuichi Izumi ${ }^{4}$ and Mitsuaki Isobe ${ }^{3}$
}

\begin{abstract}
:
Objective Tooth loss is an irreversible condition that reflects the end-stage of oral diseases, including periodontitis. Although periodontitis is a major factor in the progression of diabetes mellitus (DM) and cardiovascular disease (CVD), no previous studies have compared tooth loss in CVD patients with and without DM. Methods The subjects included CVD patients with $(\mathrm{n}=94)$ and without $(\mathrm{n}=145)$ DM who attended Tokyo Medical and Dental University Hospital. Blood examinations and periodontal measurements were performed. Results The oral and periodontal examinations revealed that the numbers of missing teeth in the DM group were increased in comparison to the non-DM group. There was no significant difference between the groups with regard to the incidence of edentulism, the probing pocket depth, the clinical attachment level or the incidence of bleeding on probing.

Conclusion We showed that the numbers of missing teeth among CVD patients with DM was significantly higher than that among CVD patients without DM.
\end{abstract}

Key words: periodontal disease, bacteria, diabetes mellitus, hemoglobin A1c

(Intern Med 57: 777-782, 2018)

(DOI: 10.2169/internalmedicine.9578-17)

\section{Introduction}

Periodontitis is a common oral inflammatory disease and is characterized by the destruction of alveolar bone and supporting connective tissue (1). The modulation of host factors, such as diabetes mellitus (DM), has been shown to be highly associated with the progression of periodontitis $(2,3)$. Although the pathogenesis of periodontitis in DM remains unclear, evidence suggests that periodontal destruction may result from prolonged hyperglycemia and that it may occur with high frequency and severity in patients with poor glycemic control (4-6). Several studies have also suggested that periodontal infection may be a risk factor for cardiovascular disease (CVD) $(7,8)$. Tooth loss is a marker for an increased risk of CVD and all-cause mortality (9). Even a few missing teeth may indicate an increased risk of CVD, DM or all-cause mortality (10). Tooth loss is an irreversible condition that reflects the end-stage of oral diseases such as periodontitis (11).

DM patients with severe periodontal disease may be particularly susceptible to microvascular and macrovascular

\footnotetext{
${ }^{1}$ Division of Periodontology, Department of Oral Interdisciplinary Medicine, Graduate School of Dentistry, Kanagawa Dental University, Japan, ${ }^{2}$ Department of Advanced Clinical Science and Therapeutics, The University of Tokyo, Japan, ${ }^{3}$ Department of Cardiovascular Medicine, Tokyo Medical and Dental University, Japan, ${ }^{4}$ Department of Periodontology, Graduate School of Medical and Dental Sciences, Tokyo Medical and Dental University, Japan, ${ }^{5}$ Department of Periodontology, Kyushu Dental University, Japan, ${ }^{6}$ Department of Oral Microbiology, Tsurumi University, Japan and ${ }^{7}$ Department of Cardiovascular Medicine, The University of Tokyo, Japan

Received: May 27, 2017; Accepted: July 3, 2017; Advance Publication by J-STAGE: November 20, 2017
}

Correspondence to Dr. Jun-ichi Suzuki, junichisuzuki-circ@umin.ac.jp 
complications, which are primarily responsible for the increased morbidity and mortality associated with DM. However, there have been few reports on the association between tooth loss or periodontitis and DM among CVD patients. We hypothesized that the presence of DM among CVD patients affected the periodontal condition and that periodontal destruction led to tooth loss. The purpose of the present study was to compare the numbers of lost teeth and the periodontal condition between CVD patients with and without DM.

\section{Materials and Methods}

\section{Study population}

Male subjects between 69 and 80 years of age were recruited from patients of the Department of Cardiovascular Medicine in Tokyo Medical and Dental University Hospital from May 2012 to August 2015. Only male patients were recruited in order to prevent gender differences from affecting results. Two hundred thirty-nine subjects were enrolled in this study. Patients who did not provide their consent for participation in this study or who had a history of other infections (or current infections) were excluded. The Ethics Committee of the School of Medicine and the School of Dentistry, Tokyo Medical and Dental University approved the protocol of the present study, and the protocol conformed to the Helsinki Declaration of 1975, as revised in 2013. Written informed consent was obtained from each subject.

\section{Medical examination}

In each case, the patient's medical history was recorded and a physical examination was performed. The subjects who were diagnosed and treated in the department for DM, hypertension (HT) and dyslipidemia (DL) were recorded. The history of cigarette smoking was obtained from an interview. Peripheral blood samples were collected, centrifuged at $1,500 \times g$ for 20 minutes and then stored until use at $-20^{\circ} \mathrm{C}$. The concentrations of low density lipoprotein (LDLc), high density lipoprotein (HDL-c), triglyceride (TG), hemoglobin A1c (HbA1c) and C-reactive protein (CRP) were measured.

DM was defined based on the following conditions: (i) fasting blood sugar $>120 \mathrm{mg} / \mathrm{dL}$, (ii) $75 \mathrm{~g}$ oral glucose tolerance test $>200 \mathrm{mg} / \mathrm{dL}(2 \mathrm{~h}$ ), (iii) blood sugar at any time point $>200 \mathrm{mg} / \mathrm{dL}$ or (iv) HbA1c $>6.5 \%$ (National Glycohemoglobin Standardization Program: NGSP). Systemic HT was defined by a systolic blood pressure of $>140 \mathrm{mmHg}$ and/or a diastolic blood pressure of $>90 \mathrm{mmHg}$. DL was defined as (i) LDL-c $>140 \mathrm{mg} / \mathrm{dL}$, (ii) $\mathrm{HDL}-\mathrm{c}<40 \mathrm{mg} / \mathrm{dL}$, or (iii) $\mathrm{TG}>150 \mathrm{mg} / \mathrm{dL}$. Obesity was defined as a body mass index of $>25 \mathrm{~kg} / \mathrm{m}^{2}$.

\section{Clinical periodontal examination}

A clinical periodontal examination was performed by three periodontists (N. Ao., N. K. and T. H) who were certified by the Japanese Society of Periodontology. The number of teeth was counted and the probing pocket depth (PPD), clinical attachment level (CAL) and bleeding on probing (BOP) were recorded at six points (buccal-mesial, midbuccal, buccal-distal, lingual-mesial, mid-lingual, lingualdistal) on a right upper molar, an upper incisor, a left upper molar, a right lower molar, a lower incisor and a left lower molar with a manual probe (PCP-UNC 15, Hu-Friedy, Chicago, USA). When the representative tooth was missing, the next tooth was used.

\section{Bacterial detection}

Unstimulated saliva was obtained. Bacterial DNA was extracted from $200 \mu \mathrm{L}$ saliva using a DNeasy Blood and Tissue kit (Qiagen, Tokyo, Japan) according to the manufacturer's instructions. A real-time polymerase chain reaction (PCR) was performed to detect three periodontopathic bacteria: Porphyromonas gingivalis, Aggregatibacter actinomycetemcomitans and Prevotella intermedia. The real-time PCR was performed according to previously described methods (12). The specific primers for each bacterium have been described previously (13). The bacterial counts in saliva were shown as count $/ \mathrm{mL}$.

\section{Antibody measurement}

Serum samples were analyzed to detect IgG antibodies against three major periodontal pathogens, $P$. gingivalis, A. actinomycetemcomitans and $P$. intermedia, using an enzymelinked immunosorbent assay as previously described (14). The absorbance of wells was read using a microplate reader at $450 \mathrm{~nm}$ with a $650 \mathrm{~nm}$ reference wavelength. Individual serum antibody levels were calculated from the standard curve obtained from the gradual dilutions of the reference and were expressed as Units/mL.

\section{Data analysis}

Numerical data were presented as the mean \pm standard deviation (SD). Student's $t$-test was performed to compare age, body mass index, CRP, LDL-c, HDL-c, TG, HbA1c, the number of missing teeth, PPD, CAL and BOP. The chisquared test was performed to compare the rate of smoking, presence of HT, DL and obesity, and the rate edentulism. Bacterial counts and antibody levels were presented as the median and 25th and 75th percentiles. The Wilcoxon test was used to compare the bacterial count and the presence of bacterial antibodies. The JMP software program (version 9.0.3; SAS Institute, Cary, USA) was used for all of the statistical analyses. $p$ values of $<0.05$ were considered to indicate statistical significance.

\section{Results}

\section{The characteristics of the study population}

The characteristics of the patients in the present study are 
Table 1. The Characteristics of the Subjects.

\begin{tabular}{lccc}
\hline Group & DM & Non-DM & $\mathrm{p}$ \\
\hline Number & 94 & 145 & \\
Age & $74.1+/-2.9$ & $73.7+/-3.5$ & 0.3407 \\
Smoker [\%] & 55 & 57 & 0.8513 \\
Hypertension [\%] & 78 & 73 & 0.4252 \\
Dyslipidemia [\%] & 54 & 40 & 0.0306 \\
Obesity [\%] & 23 & 15 & 0.1119 \\
Body mass index [kg/m²] & $24.2+/-3.5$ & $23.5+/-2.6$ & 0.0808 \\
CRP [mg/dL] & $0.67+/-2.08$ & $0.33+/-0.66$ & 0.0637 \\
LDL-c [mg/dL] & $93.2+/-25.4$ & $102.3+/-26.5$ & 0.0102 \\
HDL-c [mg/dL] & $51.4+/-14.4$ & $57.1+/-16.8$ & 0.0082 \\
TG [mg/dL] & $135.0+/-68.6$ & $132.3+/-91.0$ & 0.8129 \\
HbA1c [\%] & $6.8+/-0.8$ & $5.7+/-0.4$ & $<0.0001$ \\
\hline
\end{tabular}

DM: diabetes mellitus, CRP: C-reactive protein, LDL-c: low density lipoprotein, HDL-c: high density lipoprotein, TG: triglyceride, HbAlc: hemoglobin A1c

Table 2. The Oral Condition of the Subjects.

\begin{tabular}{lccc}
\hline Group & DM & Non-DM & $\mathrm{p}$ \\
\hline Missing Teeth & $16.1+/-9.6$ & $13.6+/-9.3$ & 0.0492 \\
Edentulous [\%] & 12 & 8 & 0.3845 \\
PPD [mm] & $2.59+/-0.65$ & $2.51+/-0.61$ & 0.4184 \\
CAL [mm] & $3.43+/-1.11$ & $3.34+/-1.14$ & 0.5926 \\
BOP [\%] & $21+/-22$ & $17+/-20$ & 0.2736 \\
\hline
\end{tabular}

DM: diabetes mellitus, PPD: probing pocket depth, CAL: clinical attachment level, BOP: bleeding on probing

shown in Table 1. There were no significant differences in the age, the rates of smoking, HT, obesity, or the body mass index values of the DM and non-DM groups. The rate of DL was higher and the HDL-c levels were lower in the DM group than they were in the non-DM group. The level of LDL-c in the DM group was lower than that in the non-DM group. The CRP levels of the two groups were comparable.

The cardiovascular diseases that were diagnosed in the two groups included: myocardial infarction and/or angina pectoris $(n=131)$ (DM group, $n=68$; non-DM group, $n=63)$, arrhythmia, $n=130$; (DM group, $n=40$; non-DM group, $n=$ 90); heart failure, $n=37$ (DM group, $n=14$; non-DM group, $\mathrm{n}=23$ ), and 34 valvular disease (DM group, $\mathrm{n}=8$; non-DM group, $n=26$ ). In cases in which a subject had multiple cardiovascular diseases, each disease was counted.

\section{Oral and periodontal conditions}

The oral and periodontal conditions of the subjects in each group are shown in Table 2. The number of missing teeth in the DM group was increased in comparison to the non-DM group. There were no significant differences in the rates of edentulism, PPD, CAL or BOP between the groups.

We also measured the bacterial counts in saliva and serum antibody levels against $P$. gingivalis, A. actinomycetemcomitans and $P$. intermedia. There were no significant differences between the groups with regard to any of the salivary bacterial counts or serum antibody levels (Table 3). We per- formed a sub-analysis of the bacteria and antibody levels in edentulous patients. No significant difference was observed in the salivary bacterial counts or the serum antibody levels of the edentulous participants (Table 4).

Table 5 shows the results of the multiple logistic regression analyses to assess the associations between DM and obesity and tooth loss. We found that many of the patients with DM had lost more than 10 teeth. After adjustment for obesity, the significant relationship remained.

\section{Discussion}

\section{Tooth loss represents an irreversible end-stage oral condition}

This cross-sectional study was conducted to reveal the relationship between DM and periodontitis in patients with CVD. Our hypothesis was that periodontitis and diabetes mellitus affect each other and influence the development of CVD. A strong relationship between periodontitis and DM has been proven (3-6) and both diseases are associated with CVD $(8,10)$. However, we had no information on whether the oral condition and periodontitis of CVD patients with DM patients was worse in comparison to patients with CVD alone. Thus, we conducted the present cross-sectional study.

In this study, we demonstrated that the number of lost teeth in the DM group was increased in comparison to the non-DM group among patients with CVD. Periodontitis is known to be the most critical cause of tooth loss in the Japanese adult population. Although there are other periodontal measurements (e.g. PPD, CAL and BOP), these measurements reflect ongoing and reversible oral conditions. In contrast, the number of missing teeth indicates irreversible end-stage oral conditions, including periodontitis. Thus, the number of missing teeth can be a hard endpoint to use in the assessment of the oral status (11). The observation in the present study indicated that DM patients with CVD had irreversible end-stage periodontitis. 
Table 3. The Detected Bacteria and Antibody Levels.

\begin{tabular}{lccc}
\hline Group & DM & Non-DM & $\mathrm{p}$ \\
\hline $\begin{array}{l}P \text {. gingivalis count } \\
\text { [counts/mL] }\end{array}$ & $\begin{array}{c}2.0 \times 10^{4}\left(5.2 \times 10^{2},\right. \\
\left.1.2 \times 10^{6}\right)\end{array}$ & $\begin{array}{c}7.5 \times 10^{4}\left(3.2 \times 10^{2},\right. \\
\left.1.3 \times 10^{6}\right)\end{array}$ & 0.6503 \\
$\begin{array}{l}\text { A. actinomycetemcomitans } \\
\text { count [counts/mL] }\end{array}$ & $0(0,0)$ & $0(0,0)$ & 0.8877 \\
$\begin{array}{l}P \text {. intermedia count } \\
\text { [counts/mL] }\end{array}$ & $0(0,0)$ & $0\left(0,4.4 \times 10^{2}\right)$ & 0.3269 \\
$\begin{array}{l}P \text {. gingivalis } \text { antibody } \\
{[\text { Units/mL] }}\end{array}$ & $156(85,331)$ & $157(64,417)$ & 0.6989 \\
$\begin{array}{l}\text { A. actinomycetemcomitans } \\
\text { antibody [Units/mL] }\end{array}$ & $62(25,102)$ & $59(30,135)$ & 0.5218 \\
$\begin{array}{l}P \text { intermedia } \text { antibody } \\
{[\text { Units/mL] }}\end{array}$ & $401(175,777)$ & $434(259,928)$ & 0.1975 \\
\hline
\end{tabular}

Data was presented as median with 25 and 75 percentiles. DM: diabetes mellitus, $P$. gingivalis: Porphyromonas gingivalis, A. actinomycetemcomitans: Aggregatibacter actinomycetemcomitans, $P$. intermedia: Prevotella intermedia

Table 4. The Detected Bacteria and Antibody Levels in the Edentulous Patients.

\begin{tabular}{lccc}
\hline Group & DM & Non-DM & $\mathrm{p}$ \\
\hline $\begin{array}{l}P \text {. gingivalis count } \\
\text { [counts/mL] }\end{array}$ & $\begin{array}{c}8.3 \times 10^{2}\left(1.2 \times 10^{2},\right. \\
\left.4.7 \times 10^{3}\right)\end{array}$ & $2.7 \times 10^{2}\left(0,3.4 \times 10^{2}\right)$ & 0.0928 \\
$\begin{array}{l}\text { A. actinomycetemcomitans } \\
\text { count [counts/mL] }\end{array}$ & $0\left(0,6.1 \times 10^{2}\right)$ & $0(0,0)$ & 0.4198 \\
$\begin{array}{l}P . \text { intermedia } \text { count } \\
{[\text { counts/mL] }}\end{array}$ & $0(0,0)$ & $0(0,0)$ & 1.0000 \\
$\begin{array}{l}P \text { gingivalis } \text { antibody } \\
{[\text { Units/mL] }}\end{array}$ & $133(40,321)$ & $54(37,142)$ & 0.2545 \\
$\begin{array}{l}\text { A. actinomycetemcomitans } \\
\text { antibody [Units/mL] }\end{array}$ & $45(20,136)$ & $36(27,60)$ & 0.5949 \\
$P$. intermedia antibody & $277(155,686)$ & $385(282,629)$ & 0.3233 \\
{$[$ Units/mL] } & & & \\
\hline
\end{tabular}

Data was presented as median with 25 and 75 percentiles. DM: diabetes mellitus, $P$. gingivalis: Porphyromonas gingivalis, A. actinomycetemcomitans: Aggregatibacter actinomycetemcomitans, P. intermedia: Prevotella intermedia

Table 5. The Relationship between Diabetes Mellitus and the Number of Patients who Lost More than 10 Teeth.

\begin{tabular}{lccc}
\hline Group & DM & Non-DM & $\mathrm{p}$ \\
\hline Crude ORs & $1.82(1.07-3.11)$ & 1 (Ref) & 0.0261 \\
*Adjusted ORs & $1.78(1.05-3.07)$ & 1 (Ref) & 0.0337 \\
\hline
\end{tabular}

*Adjusted for obesity. DM: diabetes mellitus, OR: odds ratio

\section{DM and tooth loss}

Many reports have shown a relationship between DM and periodontal disease $(15,16)$. It is widely known that DM patients develop periodontitis at a higher rate than subjects without DM (3) and that periodontal disease is an independent predictor of incidental DM (17). Because periodontitis is one of the main causes of tooth loss, it makes sense that DM patients are at a higher risk of tooth loss and edentulism than adults without DM (18). It is widely accepted that patients with DM have considerable cardiovascular risk (19) and studies have supported an association between periodontal disease and CVD, independent of known confounders, including DM (8). Thus, DM and periodontal disease can interact adversely and influence the development of CVD events. We assessed the oral and periodontal condition of DM and non-DM subjects in this study. We found that CVD patients with DM had an increased number of lost teeth in comparison to patients with CVD alone. A multiple logistic regression analysis was performed to assess the interaction between DM and obesity with regard to tooth loss. We found that a large number of DM patients had lost more than 10 teeth regardless of the presence of obesity (Table 5). The bacterial counts in the saliva and the levels of serum antibodies against periodontal bacteria were measured. However, we did not detect any significant difference in the salivary bacterial counts and serum antibody levels. Although the bacterial counts and antibody levels among edentulous patients were similar in both DM and non-DM patients, the antibody levels against $P$. gingivalis were quite high in edentulous patients with DM. Most edentulous patients must 
have suffered from periodontal infection for a long period and might have lost their teeth due to periodontitis. Further longitudinal clinical studies of large populations and experiments using animal models will be needed to clarify the interactive effects of DM and periodontal disease on CVD.

\section{Study limitations}

We showed that CVD patients with DM lost more teeth in comparison to CVD patients without DM. Although periodontal disease is a major reason for tooth extraction in adults, the reasons for tooth loss are not limited to periodontitis. Other conditions, including caries and fracture may result in tooth extraction in the clinical setting. As shown in Table 2, in addition to the number of missing teeth, the average PPD and CAL tended to be increased in the DM group in the present study. However, we have to consider that the number of missing teeth does not clearly reflect periodontal disease alone.

In the present study, we showed a significant difference in the incidence of DL. The incidence of DL among DM was higher in comparison to patients without DM; however, the LDL-c levels of the DM group were lower than those of the non-DM group. This suggested that DL was appropriately treated in the DM group. Thus, periodontitis may not affect DM via DL. The study was also associated with other limitations, including the small number of participants, the single cross-sectional study design, the limited generalizability and the limited information on other important confounding factors. A further analysis is needed to clarify the causal relationships among the factors.

\section{Conclusion}

We revealed that CVD patients with DM lost more teeth in comparison to CVD patients without DM. Further investigations are needed to reveal the detailed causal relationship between DM and periodontitis in the near future.

The authors state that they have no Conflict of Interest (COI).

\section{Financial Support}

This work was supported by JSPS KAKENHI Grant Numbers (JP25870198, JP15K20616, and JP16H05824), Ministry of Education, Culture, Sports, Science and Technology of Japan, Mitsui Life Insurance Research Foundation, Mitsui Sumitomo Marine Welfare Research Foundation, Geriatric Dental Research Foundation, Human Health Future Research Foundation, St. Luke's Hospital Research Foundation, Health Management Foundation, Taiyo Life Insurance Research Foundation, The 8020 Promotion Foundation, Terumo Science Foundation, Pfizer Health Research Foundation, General Health Promotion Foundation, Suzuken Memorial Foundation, Health Science Center Foundation, Kobayashi International Scholarship Foundation, Banyu Life Science Foundation International, and Hakujikai Institute of Gerontology Foundation.

\section{References}

1. Pihlstrom BL, Michalowicz BS, Johnson NW. Periodontal diseases. Lancet 366: 1809-1820, 2005.

2. Shlossman M, Knowler WC, Pettitt DJ, Genco RJ. Type 2 diabetes mellitus and periodontal disease. J Am Dent Assoc 121: 532-536, 1990.

3. Nelson RG, Shlossman M, Budding LM, et al. Periodontal disease and NIDDM in Pima Indians. Diabetes Care 13: 836-840, 1990.

4. Taylor GW, Burt BA, Becker MP, et al. Non-insulin dependent diabetes mellitus and alveolar bone loss progression over 2 years. J Periodontol 69: 76-83, 1998.

5. Taylor GW, Burt BA, Becker MP, et al. Severe periodontitis and risk for poor glycemic control in patients with non-insulindependent diabetes mellitus. J Periodontol 67: 1085-1093, 1996.

6. Tsai C, Hayes C, Taylor GW. Glycemic control of type 2 diabetes and severe periodontal disease in the US adult population. Commun Dent Oral Epidemiol 30: 182-192, 2002.

7. Genco R, Offenbacher S, Beck J. Periodontal disease and cardiovascular disease: epidemiology and possible mechanisms. J Am Dent Assoc 133 (Suppl): 14S-22S, 2002.

8. Lockhart PB, Bolger AF, Papapanou PN, et al. Periodontal disease and atherosclerotic vascular disease: does the evidence support an independent association?: a scientific statement from the American Heart Association. Circulation 125: 2520-2544, 2012.

9. Joshy G, Arora M, Korda RJ, Chalmers J, Banks E. Is poor oral health a risk marker for incident cardiovascular disease hospitalisation and all-cause mortality? Findings from 172630 participants from the prospective 45 and Up Study. BMJ Open 6: e012386, 2016.

10. Liljestrand JM, Havulinna AS, Paju S, Männistö S, Salomaa V, Pussinen PJ. Missing teeth predict incident cardiovascular events, diabetes, and death. J Dent Res 94: 1055-1062, 2015.

11. Hujoel PP, DeRouen TA. A survey of endpoint characteristics in periodontal clinical trials published 1988-1992, and implications for future studies. J Clin Periodontol 22: 397-407, 1995.

12. Suzuki J, Imai Y, Aoki M, et al. Periodontitis in cardiovascular disease patients with or without Marfan syndrome -A possible role of Prevotella intermedia. PLoS One 9: e95521, 2014.

13. Maeda H, Fujimoto C, Haruki Y, et al. Quantitative real-time PCR using TaqMan and SYBR Green for Actinobacillus actinomycetemcomitans, Porphyromonas gingivalis, Prevotella intermedia, tetQ gene and total bacteria. FEMS Immunol Med Microbiol 39: 81-86, 2003.

14. Ueno M, Izumi Y, Kawaguchi $Y$, et al. Prediagnostic plasma antibody levels to periodontopathic bacteria and risk of coronary heart disease. Int Heart J 53: 209-214, 2012.

15. Lalla E, Papapanou PN. Diabetes mellitus and periodontitis: a tale of two common interrelated diseases. Nat Rev Endocrinol 7: 738748, 2011.

16. Borgnakke WS, Ylöstalo PV, Taylor GW, Genco RJ. Effect of periodontal disease on diabetes: systematic review of epidemiologic observational evidence. J Periodontol 84 (4 Suppl): S135S152, 2013.

17. Demmer RT, Jacobs DR Jr, Desvarieux M. Periodontal disease and incident type 2 diabetes: results from the First National Health and Nutrition Examination Survey and its epidemiologic follow-up study. Diabetes Care 31: 1373-1379, 2008.

18. Patel MH, Kumar JV, Moss ME. Diabetes and tooth loss: an analysis of data from the National Health and Nutrition Examination Survey, 2003-2004. J Am Dent Assoc 144: 478-485, 2013.

19. Libby P, Plutzky J. Inflammation in diabetes mellitus: role of peroxisome proliferator-activated receptor-alpha and peroxisome proliferator-activated receptor-gamma agonists. Am J Cardiol 99: 27B-40B, 2007. 
The Internal Medicine is an Open Access article distributed under the Creative Commons Attribution-NonCommercial-NoDerivatives 4.0 International License. To view the details of this license, please visit (https://creativecommons.org/licenses/ by-nc-nd/4.0/).

(C) 2018 The Japanese Society of Internal Medicine

Intern Med 57: 777-782, 2018 